\title{
Scientific View in Grey System Theory
}

\author{
Xintao Xia ${ }^{1}$ \\ ${ }^{1}$ College of Mechatronical Engineering, Henan University of Science and Technology, Luoyang, China \\ Correspondence: Xintao Xia, 63\#, Xiyuan Campus, 48 Xiyuan Road, Luoyang 471003, China. E-mail: \\ xiaxt1957@163.com,xiaxt@mail.haust.edu.cn
}

Received: March 2, 2012

Accepted: March 27, 2012 Published: July 1, 2012

doi:10.5539/ass.v8n8p103

URL: http://dx.doi.org/10.5539/ass.v8n8p103

\begin{abstract}
The contribution of grey system theory to science and scientific view is discussed in three aspects of concise description for nature, harmony and unity of rationality and irrationality, and dynamic and changeable simulation and forecast for natural phenomena. A new concept of grey scientific view is proposed to reveal grey evolution process of science and scientific view.
\end{abstract}

Keywords: science, scientific view, grey scientific view, evolution, grey system theory

\section{Introduction}

Scientific development causes scientific view revolution while the latter drives the former. As a matter of fact, much new content about science and scientific view could be perceived in scientific experiment and theory research. At the beginning of 1980s, Prof. Deng Julong proposed grey system theory (Deng, 1989) to research mainly the uncertainty systems with poor information. The systems can be divided into three types: black system, white system, and grey system. The black system is not perceived one, the white system is perceived one, and the grey system is partly perceived and unascertained system between black and white systems. The relationship between grey system theory and nature, science, and scientific view could be seen from three types of the systems and meanwhile science and scientific view are perceived again from grey system theory.

\section{Description and Recognition for Nature World}

Grey system theory could concisely and profoundly describe and recognize many phenomena that scientific view could not.

In the research for scientific problems, various natural phenomena could be seen as grey, including known, unknown, or unascertained information. In scientific observation and experiments, many systems show up poor data and information and have unascertained grey problems.

For scientific view, determinism, randomization, and chaos theory are known as three great theories, almost taking the shape of three divided areas. However, object abundance and ontology seclusion, limited cognitive ability of subject, and imperfect logic result in that there is not absolute scientific view (Zhou, 2005), because the scientific history is hypothetical history originally.

For example, determinism, randomization, and chaos theory still could not perfectly explain various grey uncertainty problems in the nature, because from theory of knowledge, determinism loads excessive assumed condition in perfect state; randomization demands for excessive data and probabilistic information; and chaos theory redundantly depends on nonlinear equation and various computational procedures which seem accurate and known. The premised assumption for cognition implies assumption for cognition conclusion. Just as in statistics, the deduction result error occurred by the assumed probability distribution for statistics school may not be less than that occurred by creating prior distribution for Bayes statistics school.

Grey system theory could describe scientific problems to certain extent which are difficult, even not able to be described by determinism, randomization, and chaos theory. The methods proposed by grey system theory are unique and effective.

As a matter of fact, the description and recognition for the natural phenomena contains the description and recognition for their rules. Grey system theory shows great concern with certain (generally little) information presented in the system rather to pursue for probability distribution (known and unknown distribution) of a 
population. It has features to reveal status and evolution process of various phenomena under little data and information background.

To observe a complicated scientific phenomenon, the observation data is always fluctuant and seems to be disorganized. In fact, certain rule is implied in it. If there is much data, the randomization describing its content borrows probability distribution concept while the chaos theory borrows fractal concept. In grey system theory, the concept used is the most concise AGO (accumulated generating operation) and I-AGO (inverse AGO).

AGO refers that the each data seems to have no rule, but the obvious exponent law could be seen by cumulating data one by one, thus grey simulation and predication can be conducted. According to grey system theory, the randomness of observation data does not mean that scientific phenomenon is random. Through special data pretreatment, many natural phenomena could be discovered to imply exponent law, which could be described and recognized by grey exponential law. In grey space, much ordered and random natural phenomena could grey orderly and can therefore be recognized.

AGO describes natural phenomena from random into order change and I-AGO describes natural phenomena from order into random change. Here some contradictions to describe common laws in nature with entropy and self-organization concepts can be resolved.

Much research, such as, symmetry and asymmetry, deterministic event and random event, is to repair a defect of explanation of the natural system change by using the second law of thermodynamics: Some systems development is to increase entropy and reduce order while other systems development is to reduce entropy and increase order. The famous dissipative structure theory thinks that the different systems have different evolution ways. Consequently, this problem is resolved.

This problem could also be resolved with the development coefficient in grey system theory. In modeling grey system, the development coefficient has a capacitable area that is related to specific system attribute. If development coefficient is in the capacitable area, the grey exponential law accurately describes the system towards evolution to reduce entropy and increase order; otherwise, the system will increase entropy and reduce order. Moreover, unequal interval grey predication theory for catastrophe also describes the historical track of new system birth and old system fading. The best example is that for catastrophe phenomenon in nature, grey system theory contains many thoughts of determinism, randomization, chaos theory, and dissipative structure theory, but the grey exponential law of the grey system requires much less information. In grey system theory, stage ratio and smooth ratio are also used to describe and realize specific change details of natural phenomena, for example, to describe the influence of the severe extent of certain factor change of the weather on the recent and future climate, etc.

Upon the least observation information, the grey exponential law could reveal current and future status of natural phenomena and could concisely and clearly recognize all systems. It can be seen from grey system theory that the simple grey exponential law is the concise and deep description and cognition for natural phenomena. From this point, the significance of grey system theory in the aspect of scientific view is mainly that science could reflect the objective rules in the nature and nature status, and general evolution rule; that science is the complicated theory knowledge system, concept, and method to be expressed simplest; and that limited communication among determinism, randomization, and chaos theory is put into practice in a sense.

\section{Unity of Rationality and Irrationality}

The rationality and irrationality in science can be united in grey system theory.

Great attentions have been given to the problems that science is rational or irrational in scientific view for reason that the inherent characteristics of the science is finally discovered and that the long-standing rationalism tradition in western science beautifies excessively science. Induction is only the association of people's mentality habit without logical proof, which could not have bad influence on scientific reputation. As a matter of fact, it has been indicated by the scientific development history that science has both rational factors and irrational factors, because science is in the society. In the science, the rational factors and irrational factors have been discriminated and united. The integral whole for this pair of contradiction powerfully promotes the progress and development of science and this is the greatness of science, so, science deserves for people's respect and attracts people's struggle for it in his entire life. The discovery of irrationalism, just like rationalism, has its inherent logicality and indicates development of philosophy of science.

Probability distribution arrangement in classic statistics, prior distribution of Bayes statistics, and membership function of fuzzy set theory (Xia, 2000), even the conditional assumption of rational linear and nonlinearity theory still contain irrational factors. In grey system theory, there are the contribution problems of rational and 
irrational factors to science.

In grey predication, the stage ratio of grey modeling series must be implemented into the required feasible domain, otherwise data pretreatment is required. There are many methods for data pretreatment. The experience disposal methods are recommended in grey system theory such as translation, logarithm, and square root, etc and the specific application is chosen based on experience. The error of description for research object has certain relationship with experience. However, the error of description for research object will become greater if there is not intervention of those irrational factors, even distorts features of research object, generating research results without scientific significance.

Obviously, the irrational factors defeat rational factors, revealing that rationalism is unable or temporarily unable to reveal current status and evolution process of natural phenomena.

In fact, irrational factors are everywhere in statistics and chaos theory research. For example, the numerical evaluation of some simultaneous differential equations is impossible to be solved without manual intervention for parameter introduction and adjustment.

Seen from scientific view, any method is effective but not absolute. Irrational method may not be the best one but the best or worst standard is not decided by the method, which may be related to the specific research object, because objective existence does not fit well with scientific discovery.

Science is a kind of human behavior. It is not only the observation and deduction formula, but also mainly a kind of way to dispose the experience. The word of "science" may be proper as a verb than a noun. If someone is said to be making science, actually he disposes the experience based on certain assumption and technology. From this significance, scientific thinking method becomes more important (Li, 2002).

However, the so-called scientific view of "science is not truth, not progressive, and nor highly confirmed" ignores the fact that some "irrational factors" involved in scientific decision-making could be effectively controlled and reduced in the analysis of other "irrational factors". The effect of autonomy and automaticity is better in non-statistics theory research (Xia, 2010) based on grey system theory than in statistics and determinism research.

Obviously, the significance of grey system theory in scientific view aspect could be embodied in that rationality and irrationality are not completely opposite; that the comparison of the two relies on specific condition and object without comparison in general meaning; and that sometimes, the two are mutually mixed together and enhanced to get highly unity, continuously enriching and developing science.

\section{Science in Evolution Process}

It is forecasted that science is not static but in changeable evolution in grey stimulation and prediction process.

Scientific theory, scientific experiment, and scientific calculation have played an important position in science. It is indicated by the research that scientific calculation can result in scientific revolution, because scientific theory is an arithmetic system and scientific law is computationalism scientific view of algorithm (Hao, 2005). Of course, it relies on digitization and numeralization of knowledge. In grey system theory, this view has far-reaching significance.

From Newtonian mechanics to quantum mechanics and to nonlinearity dynamic mechanics, the calculation method change results in science from static state to dynamic state, from single to multidirectional development, and from isolate to correlative development. The outstanding nonlinear dynamics splendidly depicts the colorful natural world including chaos, fractal, and attractor, etc. Among that an important discovery is that the certain system will show uncertain information and moreover be extremely sensitive to initial value. It is not known that how many people are wondered for natural prospect repainted by chaos theory (Yu, 2005).

One point deserves for attention is that the chaos theory must adopt different simultaneous differential equations in displaying different natural phenomena. This means that fixed and changeless mathematical model is required. That is, the mathematical model for natural phenomena is invariable and cryptology is an invariable natural phenomenon, involving in wrong trap of static science and universe.

In grey system theory, the "shadow" of grey differential equation is given miraculously and this "shadow" is just form in mathematics, having nothing to do with specific natural phenomenon, and grey action and development coefficient depend on observation data in calculation and are changeable. It means that grey stimulation and prediction process actually is a changeable dynamic module and the change process is natural to online track and forecast natural phenomena and to correct timely error with natural phenomena. Thus, grey system theory not only reflects input and output change but also the natural phenomena are in changing process, revealing that 
science is not the end and it is evolving. Scientific evolution includes factors to influence on science and factors influenced by science and scientific system itself. This is the other scientific scene presented by grey system theory.

\section{Conclusion}

It is found from the above research for grey system theory scientific view that the content and expression of science and scientific view is too colourful to depict with color. For a moment, it is depicted with grey because grey represents not completely known and unascertained with theory and practice and all in changeable evolution. This is grey scientific view.

\section{Acknowledgements}

This project was funded by the National Natural Science Foundation of China (Grant No. 51075123), the Natural Science Research Project of the Education Department of Henan Province (Grant No. 2010B460008), and the Doctoral Scientific Research Initiation Fund of Henan University of Science and Technology (Grant No. 09001318).

\section{References}

Deng, Julong. (1989). Introduction to grey system theory. The Journal of Grey System, 1(1), 1-24.

Hao, Ningxiang. (2000). Change of scientific computing and scientific view. Science and Technology and Dialectic, 17(6), 6-10. (in Chinese).

Li, Yanhui. (2002). Overview of Mr. Qian Xuesen's “Thinking Science” from the angle of the systematic view. Journal of Xiangtan Normal University (Social Science Edition), 24(3), 139-141. (in Chinese).

Xia, Xintao, Lv Taomei, \& Meng, Fannian. (2010). Gray Chaos evaluation model for prediction of rolling bearing friction torque. Journal of Testing and Evaluation, 38(3), 291-300.

Xia, Xintao, Wang, Zhongyu, \& Gao, Yongsheng. (2000). Estimation of non-statistical uncertainty using fuzzy-set theory. Measurement Science and Technology, 11(1), 430-435. http://dx.doi.org/10.1088/0957-0233/11/4/314

Yu, Dagan. (2005). Cross science and the development of geoscience in the New Century. Journal of East China Institute of Technology (Social Science Edition), 24(1), 1-7, 13. (in Chinese).

Zhou, Liyun (2005). Is absolute scientific view possible?. Science of Science and Management of Science and Technology, 26(4), 5-10. (in Chinese).

\section{Remarks}

This is a postscript of the academic monograph titled Rolling Bearing Test Analysis and Assessment with Poor Information (Science Press, Beijing, 2007). 